\title{
Development of 3D models for faculty of education database system via monitor's graphical user interface interactive for perception and utilization
}

\author{
Suebpong Prabyai ${ }^{1, *}$, Yotravee Waythongkhum ${ }^{1}$, and Patiya Pattanasak $^{1}$ \\ ${ }^{1}$ Ramkhamhaeng University, Huamak, Bangkapi Bangkok, Thailand
}

\begin{abstract}
This study is a research and development study that used systematic approaches as an operational concept. The objectives of the study included: (1) To study the usage demands of an on-screen interactive Graphical User Interface (GUI) for the Faculty of Education database. (2) To create 3D models and study their efficiency in the usage of an onscreen interactive GUI for the Faculty of Education database. (3) To evaluate the perception and utilization of 3D models in the usage of an onscreen interactive GUI for the Faculty of Education database. The population in this study included; (1) 100 staff and students in the Faculty of Education - who were selected using Purposive Sampling to study usage demands of an on-screen interactive GUI for the Faculty of Education database. (2) 5 experts and 40 staff and students of the Faculty of Education - who were selected using Purposive Sampling to study the efficiency of 3D models in the usage of an on-screen interactive GUI for the Faculty of Education database. (3) 300 staff and students of Ramkhamhaeng University who used 3D models with the on-screen interactive GUI of the Faculty of Education database within the 1-month test period of the study. The research instruments used in this study were: (1) a questionnaire about usage demands of an on-screen interactive GUI for the Faculty of Education database, (2) an expert opinion evaluation form, and (3) an assessment form for perception and utilization of 3D models in the usage of an on-screen interactive GUI for the Faculty of Education database - all of which were found with .930 reliability and item discrimination values between $.71-.85$.
\end{abstract}

\section{Introduction}

Thailand has come a long way in application of advanced information and communications technology to increase the country's economic and social progress, especially in the creation of 3D model-using package software such as AutoCAD, Autodesk Revit, ArchiCAD, Solid Works, and other Virtual Reality related applications. These programs are used to create interactive graphics in the form of command input and

\footnotetext{
*Corresponding author: suebpong_p@hotmail.com
} 
feedback output which is then perceived by the users in the form of sensation and perception. This gives the users learned experience on system commands necessary for the next usage.

With computer technology also being developed to greater capabilities, software developers have access to more tools that can be used to create a user interface which is easier on the eye and easier to use, known as "Graphical User Interface (GUI)". More importantly, some GUIs also support interactions using natural languages such as voice and touch, which is convenient for a less skilled user [1]. Therefore, user interfaces for interactive multimedia are mostly a GUI which can be designed to have an attractive and interesting appearance.

As a user interface is a tool that users use to interact with a system, therefore the designers must have good knowledge of the demands and details of the users. In addition, according to the principles of Human-Computer Interaction (HCI), an effective system design that is conducive to efficient usage requires understanding of users' diversity. The design should help assist the users through the use, leave them with positive impression towards the system usage, prevent usage confusion, etc. [2] When humans are stimulated by an environment, it creates a sensation, which can be perceived via 5 sensory organs sight from eyes, hearing from ears, taste from tongue, smell from nose, and touch from skins. However, there are also 3 inner factors that help complete the process of perception, which are sensory nerves, motor nerves, and motor organs that receive sensory impulses, send responding impulses, and respond to the stimuli perceived via the 5 outer sensory organs or sensory receptors such as eyes, ears, nose, tongue, and skin. [3,4] Perception is therefore an important learning foundation for an individual, as every behavioral response is based on perception of one's environment and the ability to interpret that state. Hence, the design of an interactive GUI is another critical factor for the usage of 3D models with a database system, as it can promote perception and utilization of the system among the users.

The Faculty of Education is one of the first 4 faculties founded alongside Ramkhamhaeng University as recorded in the Act of Ramkhamhaeng University 2514 B.E. Nowadays, the Faculty of Education operates in 3 buildings - The Faculty of Education Building (EOB), Nakhon Chum Building (NCB), and Si Chum Building (SCB) - under the supervision of the board of management which includes the dean, the vice dean, and heads of department. The faculty bodies consist of 10 departments and other offices that include the Secretary Office, Administration and General Service, Storage and Supply, Educational Services, Policy and Planning, and Quality Assurance [5]. The faculty was currently found with no database with the use of 3D-based communication.

Moreover, the faculty's information, which can be categorized into general information - history, philosophy, aspiration and vision, architectural information - of the 3 buildings EOB, NCB, and SCB, administrative structure information - the board of management, faculty committees, departments, and supporting bodies, personnel information - of the dean, the vice dean, directors, heads of departments, faculty members, supervisors, and support personnel, and other supporting information was not put together as an official database of the Faculty of Education.

Thus, the main focus of this research was to create 3D models of the faculty's building and link them to the Faculty of Education database using an on-screen interactive GUI. This development can be used as a prototype or guideline for the future development and usage of 3D models in a database with an on-screen interactive GUI of other departments, schools, and sections, both inside and outside the university. The usage of the 3D models on the database's on-screen interactive GUI should also help promote data perception and encourage utilization of the data among the staff, students, lecturers, and others who are interested. 


\section{Methodology}

\subsection{Population and sample group}

The sampled population in the study consisted of 3 groups: (1) 100 staff and students in the Faculty of Education - who were selected using Purposive Sampling to study of usage demands of an on-screen interactive GUI for the Faculty of Education database. (2.1) 5 experts - who were selected using Purposive Sampling to evaluate the efficiency of 3D model usage with an on-screen interactive GUI for the Faculty of Education database, regarding 3D-model design, graphic design, and interaction design. (2.2) A total of 40 staff and students of Ramkhamhaeng University, 10 in the first experiment and 30 in the second experiment- who were selected using Purposive Sampling to study the efficiency of 3D model usage with an on-screen interactive GUI for the Faculty of Education database. (4) 300 staff and students of Ramkhamhaeng University who used 3D models with the onscreen interactive GUI of the Faculty of Education database within the 1-month test period of the study.

The research instruments used in this study were: (1) a questionnaire about usage demands of an on-screen interactive GUI for the Faculty of Education database, (2) an expert opinion evaluation form for the efficiency of 3D model usage with an on-screen interactive GUI for the Faculty of Education database - which was screened using the Item Objective Congruence (IOC) Index, and (3) an assessment form of perception and utilization of 3D models in the usage of an on-screen interactive GUI for the Faculty of Education database - which was also screened with IOC. The items of the instruments were found with acceptable quality with IOC score $=1$, reliability at .930 , and item discrimination values between $.71-.85$.

\subsection{Data analysis}

The experts' evaluation of the efficiency of 3D model usage with the on-screen interactive Graphical User Interface (GUI) for the Faculty of Education database was analyzed using IOC. On the contrary, the results from the questionnaire about usage demands of an onscreen interactive GUI for the Faculty of Education database and the assessment form of perception and utilization of 3D models in the usage of an on-screen interactive GUI for the Faculty of Education database were analyzed using descriptive statistics such as Percentage, Mean, Standard deviation.

\section{Results and discussions}

3.1 Staff and students in the Faculty of Education were found demanding the usage of the Faculty of Education database via an on-screen interactive GUI at a high level ( $\bar{X}=3.75$ ). When considered aspect by aspect, all were found demanded at a high level, with "systemuser interaction design" found to have the highest demand $(\bar{X}=3.80)$, followed by "components of the interactive GUI" ( $\bar{X}=3.77)$, "graphic presentation techniques" $(\bar{X}=$ $3.74)$ and "data access" ( $\bar{X}=3.67)$. This might be due to the fact that the Faculty of Education did not yet have any digitalized information about its buildings.

The personnel information on both lecturers and supporting staff of the Faculty of Education was also kept only at the Personnel Division and other major pieces of data were kept by the university. Therefore, staff and students were found demanding easy access to the up-to-date data. They also demanded access to a network linking the personnel database system to the graphic information of the buildings, so that staff, students, and those 
interested could utilize this information. An article [6] concluded that it is necessary for a designer to know their users - to know who they are designing for and what are the goals they aim to meet through their usage. Consideration of these factors will help determine the components necessary in the interface design for more efficient communication with the users. When considering aspect by aspect, it was found that most users demanded "systemuser interaction design" which entails the use of graphics in the place of text, implementation of animated images, creation of a homepage - that links to-and-from other pages containing information both on-and-off the database, and creation of interactive graphics - i.e. icons or symbols - for them to comprise to a graphic menu.

As for the "components of the interactive GUI", the users demanded that the components should be easy to understand, the text and background should be in light-anddark contrast colors, sans serif fonts should be used for a modern look, zoom-in clicking option and on-hover zoom should be available for images, icons should be in an appropriate easy-to-see size on the selected screen, and there should be no limit to usage time or the number of connected devices. For "graphic presentation techniques", the users demanded clean-looking graphics, use of the same formatting on every page - including the use of similar item positions, colors, font styles, menu, background colors, alignments, and tables, and different categories of data being clearly separated and presented according to the type and nature of information. As for "data access", the users demanded that they have easy access to the information, they should be able to access the data without having to go through a homepage as there should be a page for the main menu and other pages with an alternative menu, data should be divided into groups and assigned with numbers, and there should be buttons for commands such as 'next', 'back'. 'go to the first page', or 'go to the last page'. These findings were in line with the statement that $[7,8]$ designing a user interface is a process that requires communication with the users. The designers must take into account the users' diverse skills, hence it is important to start by learning about them. Users can be divided into 3 main categories based on their skill which are - novices or firsttime users, knowledgeable intermittent users, and expert frequent users. In the article [9] "Update An Older Interface" discussing computer-user interfaces, it is mentioned that designers of user interfaces must consider designing an interface that is suitable for the users, and that when designing a new user interface, it must not affect the users who are still accustomed to the older version. In the study "The user interface design for online shopping" [10], the results revealed online shopping websites require the use an interface with data links in the forms of Menu Page, Pyramid, Escape Hatch, Fat Menu, Sitemap Footer, Breadcrumbs. The websites' background should be in light color and contain no pattern. The appropriate font sizes should be between 6-14 pixels and an entire webpage should use a color palette of 3 colors.

3.2 The experts' overall evaluation of the efficiency of 3D model usage with the onscreen interactive Graphical User Interface (GUI) for the Faculty of Education database was found at the IOC score of 0.99 or an appropriate level. The 3D models of the Faculty of Education buildings were created in this study using the program Sketch Up. The Faculty of Education's architectural and personnel database was created with the programs Adobe Illustrator and Adobe Photoshop. The data links between the 3D models and the interactive GUI of Faculty of Education database - which included elements such as text, photos, pictures, 3D images, audio, videos, animation - were created using Adobe Dreamweaver and PhpMyAdmin. The components included were considered based on appropriation and real users' demands. iFrames for 3D models were created using Adobe Dreamweaver installed on the database system. Validation of interoperability between 3D models and the database, gathering of uncomplicated information, and systematic data sharing were done using Google Chrome. 
Users are granted Random Access - they can access data from any page within the system as every partition of data was designed to be connected. Hence, a user can move from one page to another without having to go back to the homepage index. According the Principles of Design for Interaction [11], designs should promote ease of learning, efficiency of use, and memorability.

They should also help minimize errors and satisfy the users. This is in line with an article [12] which mentioned that Sketch Up is a program with a 3D GUI that is designed to be easy to use, suitable for users who want to design general models without having had any experience using software for 3D models before. Moreover, a user interface for interactive multimedia using $3 \mathrm{D}$ objects, such as 3D images that allow users to navigate around them or explore their shapes, provides the users more information than $2 \mathrm{D}$ objects.

The objects are usually graphic models which are designed to be attractive and interesting and be able to communicate data between the system and users as efficiently and easily as possible to improve understanding. In a study [13] in which Sketch Up was used for buildings' shading and daylighting analysis, it was found that using models made with Sketch Up had more advantages than physical models.

This is consistent with the study "Cultural heritage interactive 3D models on the web: An approach using open source and free software" [14] in which the researcher created 3 models and linked them with graphics, descriptive data, and metadata using PHP, PostgreSQL, and the X3D which also linked them with website URLs allowing users to effectively access 3D models and their linked data within a short period of time. In the study "Utilizing Google Earth and Google Sketch Up to Visualize Wind Farms" [15,16], Google Earth and Google Sketch Up were used to demonstrate wind farms.

The programs allowed users to see a wind farm from different angles with background in several places as 3D models can be created with Google Sketch Up and uploaded to Google Earth. Those who are interested can see these created 3D wind farms by connecting to internet and running Google Earth.

3.3 The results of assessment for perception and utilization of 3D models in an onscreen interactive GUI of the Faculty of Education database

The assessment for perception and utilization of 3D models in an on-screen interactive GUI of the Faculty of Education database is shown in Table 1.

Table 1. Means and Standard Deviations of perception and utilization of 3D models in the on-screen interactive GUI for the Faculty of Education database.

\begin{tabular}{|l|c|c|c|}
\hline \multicolumn{1}{|c|}{ Item } & $\bar{X}$ & S.D. & Assessed Level \\
\hline Perception & $\mathbf{4 . 3 1}$ & $\mathbf{0 . 5 5 1}$ & High \\
\hline 1. Receiving up-to-date and accurate data. & 4.43 & 0.627 & High \\
\hline 2. The data is adequate and easy to understand. & 4.29 & 0.628 & High \\
\hline $\begin{array}{l}\text { 3. There is implementation of 3D models and 360- } \\
\text { degree photos. }\end{array}$ & 4.33 & 0.759 & High \\
\hline $\begin{array}{l}\text { 4. On-screen interactions make 3D models interesting } \\
\text { and convenient to use. }\end{array}$ & 4.19 & 0.720 & High \\
\hline 5. Icons, text, and images are clear and easy to see & 4.31 & 0.714 & High \\
\hline Utilization & $\mathbf{4 . 3 4}$ & $\mathbf{0 . 5 6 1}$ & High \\
\hline $\begin{array}{l}\text { 1. Being able to use the data obtained to benefit work } \\
\text { or learning. }\end{array}$ & 4.34 & 0.642 & High \\
\hline 2. The received data is up-to-date and practical. & 4.29 & 0.619 & High \\
\hline $\begin{array}{l}\text { 3. The presented information helps encourage usage } \\
\text { among staff and students. }\end{array}$ & 4.39 & 0.658 & High \\
\hline
\end{tabular}

From Table 1, it shows that the lecturers, staff, and students of the Faculty of Education assessed the overall perceptive aspects of the on-screen interactive GUI at a high level ( $\bar{X}$ 
$=4.43)$. When considered aspect by aspect, all were found demanded at a high level, with "receiving of up-to-date and accurate data" found to be at the highest level $(\bar{X}=4.31)$, followed by "There is implementation of 3D models and 360-degree photos" ( $\bar{X}=4.33)$, Icons, text, and images are clear and easy to see. $(\bar{X}=4.31)$, "The data is adequate and easy to understand." $(\bar{X}=4.29)$, and "On-screen interactions make 3D models interesting and convenient to use." $(\bar{X}=4.19)$. As for the utilization, it was overall assessed to be at a high level $(\bar{X}=4.34)$. However, when considering utilizing aspect by aspect, all of them were assessed to be at a high level, with "the presented information helps encourage usage among staff and students" found to be at the highest level $(\bar{X}=4.39)$, followed by "Being able to use the data obtained to benefit work or learning." ( $\bar{X}=4.34)$, and "The received data is up-to-date and practical" $(\bar{X}=4.29)$.

This might be because staff and students of the Faculty of Education were able to access complete and up-to-date data through the easy usage and access with the application of 3D models and 360-degree images of each building. 3D models allowed them to explore the buildings from all directions, while the 360-degree images showed details on each floor of the building, making the perception of data easier to understand.

Additionally, the easy-to-understand and adequate presentation of data, on-screen interactions, icons, text, and images that are clear and easy to see might also be what made the $3 \mathrm{D}$ models more interesting. They also helped users understand the data they perceived more, leading to more convenience in system usage and a high level of satisfaction towards the database system with this GUI. In the study "The development of user interface interactive for perception and utilization in working age (45-65 year)", it was found that users were demanding data access resembling page turning and on-screen icons with symbols and text explaining them underneath.

As for interactions, the users demanded manual controls on media. In terms of satisfaction, the users were most satisfied with being able to control media by themselves. For design aspects, icons of appropriate sizes with clear standardized definitions and usage allowing the users to easily go back to the index and other pages were found assessed at a high level.

Meanwhile, utilization was overall assessed to be at a high level $(\bar{X}=4.34)$, and when considering aspect by aspect, all of them were assessed to be at a high level, with "the presented information helps encourage usage among staff and students" at the highest level. The staff users assessed that they could use the data advantageously in their work, and that it might also help promote the Faculty of Education to be more well-known among general public through internet access.

Meanwhile the students could also benefit from some pieces of obtained data - i.e. data about the board of management, lecturers, and departments, or their contact information by utilizing them in classes or learning. All of these uses might be possible due to the nature of data presented as it was assessed as practical, up-to-date, and ready-to-use. "Development of a user interface for a learning content management system" [17,18], a study conducted based on the principles of Learning Content Management System (LCMS), was divided into 2 parts [19].

The first part was to develop user interface for the LCMS and the second part was to develop a mathematical equation tool compatible with the program's module. The results then found that the users had a high level of satisfaction towards the user interface and the mathematic tool, and were able to apply them in their school e-learning development. In another study [20,21], in which Augmented Reality (AR) technology was used to develop $3 \mathrm{D}$ interactive recommendation models for agriculture products, it was found that assessors of the AR-applied models for agricultural farms were able to satisfy business owners and general users very well. 


\section{Conclusion}

The conclusion of the research results is as follows;

1. Staff and students in Faculty of Education were found demanding the usage of the Faculty of Education database via an on-screen interactive GUI at a high level ( $\bar{X}=3.75)$. When considered aspect by aspect, all were found demanded at a high level, with "systemuser interaction design" found to have the highest demand $(\bar{X}=3.80)$

2. Efficiency of 3D model usage with the Faculty of Education database via an onscreen interactive GUI was evaluated at an appropriate level $(\mathrm{IOC}=0.99)$.

3. The results of assessment for perception and utilization of 3D models in an on-screen interactive GUI of the Faculty of Education database showed that overall perception was at a high level $(\bar{X}=4.43)$. When considering perceptive aspect by aspect, all of them were assessed to be at a high level, with "receiving of up-to-date and accurate data" found to be at the highest level $(\bar{X}=4.31)$. As for utilization, it was overall assessed to be at a high level $(\bar{X}=4.34)$, and when considering utilizing aspect by aspect, all of them were assessed to be at a high level, with "the presented information helps encourage usage among staff and students" found to be at the highest level $(\bar{X}=4.39)$.

\section{Acknowledgement}

The completion of this study could not have been accomplished without the funding from the Research and Development Institute, Ramkhamhaeng University. I would like express deep and sincere gratitude to the President of Ramkhamhaeng University, Asst. Prof. Wuthisak Lapcharoensap, as well as the specialists and experts who kindly provided advice and evaluations of the research instruments' quality until the successful completion of this project

\section{References}

1. R. Mayer, R. Moreno, J. of Educational Psychology 38(1), 43-52 (2003)

2. Inya et al., HCI \& Information (2006) http://tikkydance.exteen.com

3. A. Arkhipov et al., E-Planning and Collaboration: Concepts, Methodologies, Tools, and Applications 2-3, 799-817 (2018)

4. Microsoft Corporation, The windows interface guidelines for software design (Microsoft Press, Washington D.C., 1995)

5. Ramkhamhaeng Universiy, Faculty of Education 2020 The Management Structure of The Faculty of Education (2020) www.edu.ru.ac.th

6. D. Joiner, A summary of principles for user-interface design (2012) http://www.sylvantech.com/talin/projects/ui_design.html

7. B. Shneiderman, C. Plaisant, Design the user interface: Strategic for effective human computer interaction (Pearson Education, Boston, MA, 2005)

8. A. Tran et al., International J. of Recent Technology and Engineering 8(2 SI 11), 3883-3888 (2019)

9. M. Tell, Update and older interface. Empowering people (Addison Wesley, Seattle, WA, 1990)

10. Y. Yanyothin, The user interface design for online shopping. A Thesis for the Master of Fine Arts Program (Silpakorn Unversity, 2012) 
11. L. Baggerman, Design for interaction (Beverly, MA: Rockport Publishers, 2000)

12. N. Chaemkham, P. Bomklang, SketchUp 8 For Amateur Users - A Simple Method For Creation of Beautiful Models (Provision, Bangkok, 2012)

13. Y. Chaiyakul, Google SketchUp for shading and daylighting analysis (2008) https://arch.kku.ac.th/pr/wp-content/uploads/2011/08/Abstract36.pdf

14. A. Guarnieri et al., J. of Cultural heritage 11, 350-353 (2010)

15. H. Van et al., International J. of Energy Economics and Policy 8(4), 21-27 (2018)

16. R. Wolk, Utilizing Google earth and Google sketch up to visualize wind farms (2008) https://ieeexplore ieee.org/stamp/stamp.jsp?tp=\&arnumber=4559793

17. B. Chartchuea, The development of user interface interactive for perception and utilization in working age (45-65 year). A Thesis For the Master of Science Program (Rajamangala University of Technology Thanyaburi, 2013)

18. H. Mai et al., International J. of Recent Technology and Engineering 8(2 SI 11), 38763882 (2019)

19. M. Ihnatenko et al., International J. of Economics and Business Administration 7(2), 290-301 (2019)

20. B. Sriruengphan, Development of a user interface for a learning content management system. A Thesis For the Master of Science in Technical Education Program (King Mongkut's University of Technology North Bangkok, 2008)

21. J. Dowsai, S. Natthawutsit, The Development Of 3D Interactive Recommendation Model For Agriculture Products Using Augmented Reality Technology (2018) https://www.tci-thaijo.org/index.php/pkruscitech/article/view/154900/112643 